\title{
NOTAS
}

\section{Valoración de diámetro menor, ramas y módulo de elasticidad en trozas de Pinus radiata mediante una regresión parcial}

\author{
Valuing small end diameter, branches and modulus of elasticity in Pinus radiata \\ logs by a partial regression
}

\author{
Rosa M Alzamora ${ }^{\text {a* }}$, Cindy Y Contreras ${ }^{b}$ \\ *Autor de correspondencia: Universidad de Concepción, Facultad de Ciencias Forestales, \\ Departamento de Manejo de Bosques y Medio Ambiente, Victoria 631, Concepción, Chile, tel.: 56-41-2204905, ralzamora@udec.cl

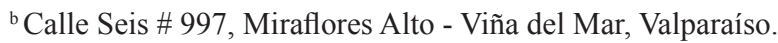

\section{SUMMARY}

Economic values of $P$. radiata wood quality traits contribute to decision making in tree breeding and log segregation by providing a guide to efficiently target those attributes possessing the highest value; however, in Chile there are not studies that report this information. Thus, the current study applied the partial regression method to value log small end diameter, branch diameter and dynamic modulus of elasticity in a sample of 71 unpruned logs. The average log conversion return was 57 US\$ ${ }^{-3}$; it showed a high and significant correlation with $\mathrm{MoE}_{\mathrm{d}}$. The regression generated values that are comparable with similar worldwide studies. Thus, economic values were $0.6 \mathrm{US} \$ \mathrm{~cm}^{-1}$ and $17.1 \mathrm{US} \$ \mathrm{GPa}^{-1}$ for small end diameter and dynamic modulus of elasticity, respectively; whereas the economic value of branch diameter was zero. Future studies to value wood quality attributes should apply partial regressions and alternative approaches to compare them, and hence, to determine which one would be the most suitable for wood production in Chile.

Key words: radiata pine, log attributes, structural lumber, partial regression.

\section{RESUMEN}

Los valores económicos de atributos de calidad de madera de $P$. radiata son una guía para tomar decisiones en mejoramiento genético y segregación de trozas, porque permiten focalizarse en los atributos con mayor valor; no obstante, en Chile, dicha información no ha sido reportada. Este estudio aplicó el enfoque de las regresiones parciales para valorar los atributos de troza: diámetro menor, diámetro de rama y módulo de elasticidad dinámico $\left(\mathrm{MoE}_{\mathrm{d}}\right)$ a partir de una muestra de 71 trozas no podadas. El retorno de conversión promedio de las trozas fue $57 \mathrm{US} \$ \mathrm{~m}^{-3}$ y mostró una positiva y significativa correlación con el $\mathrm{MoE}_{\mathrm{d}}$. La regresión generó valores comparables a

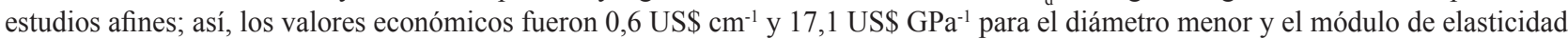
dinámico, respectivamente. El valor económico del diámetro de rama fue cero. Futuros estudios de valoración de atributos deberían aplicar y comparar las regresiones parciales con enfoques alternativos para saber cuál sería el más adecuado y costo efectivo para la producción maderera en Chile.

Palabras clave: pino radiata, atributos de troza, madera estructural, regresión parcial.

\section{INTRODUCCIÓN}

La madera de Pinus radiata D. Don es apta para una amplia gama de productos como madera estructural, madera de apariencia, tableros y papel. No obstante, cada producto tiene requerimientos específicos de calidad de madera. Zhang (1997) define calidad de madera como todos aquellos atributos de la madera que afectan la cadena de recuperación de valor y el servicio de los productos finales. En el caso de trozas de $P$. radiata, el volumen y el módulo de elasticidad dinámico han mostrado ser determinantes para explicar la recuperación de grados estructu- rales (Apiolaza 2009). Por su parte, el tamaño de rama ha mostrado un impacto negativo en la producción de madera estructural (Xu y Walker 2004). Para madera de apariencia, los atributos más importantes en trozas podadas son volumen, diámetro del cilindro con defectos y largo de internudos. En cambio, la calidad de madera para pulpa depende de la densidad básica y la composición química (Kumar 2004).

La mayoría de los atributos de $P$. radiata son heredables y factibles de potenciar con mejoramiento genético y silvicultura. No obstante, cualquiera sea la estrategia, existe la necesidad de jerarquizar dichos atributos para guiar la 
mejora de árboles de modo de producir más y mejor materia prima para satisfacer los requerimientos de calidad de los productos finales. Desde la perspectiva genética, lo anterior se logra especificando un objetivo de mejoramiento, que se define como el cambio en el resultado económico de un sistema de producción dado un cambio en el valor genético. Hazel (1943) definió el valor genético agregado como la combinación lineal de los valores genéticos aditivos de dos o más atributos, ponderados por sus respectivos valores económicos [1].

$$
H=v_{1} c_{1}+v_{2} c_{2}+\ldots+v_{n} c_{n}=v^{\prime} c
$$

Donde: $H=$ valor genético total, $v$ y $c=$ valores económicos y los atributos objetivo, respectivamente.

El modelo bioeconómico es el más utilizado para estimar " $v$ ", y consiste en un modelo que determina el cambio en rentabilidad de un sistema de producción debido al cambio en el nivel del atributo (Ivković et al. 2006). Otros métodos son las regresiones parciales (Aubry et al. 1998), los modelos hedónicos y las fronteras de eficiencia (Alzamora et al. 2013).

Las regresiones parciales relacionan los atributos de trozas con el valor de la madera producida; así, los coeficientes parciales derivados de la regresión corresponden al valor económico de los atributos. El método es sencillo de modelar e interpretar; no obstante, asume linealidad del retorno económico ante aumentos del atributo, lo cual no aplica cuando hay especificaciones de precio-calidad impuestas por umbrales de una o más características (Burdon 1990). Esta limitante se supera usando aproximaciones lineales a través de programación separable convexa y no-convexa (Tomlin 1981). Cotterill y Jackson (1985) utilizaron el método de la regresión parcial para valorar altura, diámetro, rectitud, y diámetro de rama de $P$. radiata en Australia.

El presente estudio tiene como objetivo aplicar el enfoque de las regresiones parciales para valorar atributos de calidad de madera en trozas de $P$. radiata. Las hipótesis son: a) la regresión parcial es un enfoque plausible para valorar diámetro menor, módulo de elasticidad dinámico e índice de rama, y b) los valores de los atributos son comparables a los reportados por estudios afines.

\section{MÉTODOS}

Una base de 71 segundas y terceras trozas, no podadas de $5 \mathrm{~m}$ de largo, provenientes de dos rodales de $P$. radiata (26 y 28 años) fue facilitada por Solid Wood Innovation ${ }^{1}$. Ambos rodales estaban en Kaingaroa Timberland (Nueva Zelandia). El rodal 1 se plantó en 1979, estaba podado hasta la altura de $4 \mathrm{~m}$ y su densidad final fue 158 árboles ha ${ }^{-1}$; su suelo pertenece a la familia Te Rangiitaf. El rodal 2 fue

Solid Wood Innovation (SWI) es un consorcio neozelandés de investigación (http://wqi.co.nz/). plantado en 1981 y su densidad final fue 268 árboles ha ${ }^{-1}$ con una altura de poda de $6,9 \mathrm{~m}$; su suelo corresponde a la familia Taraweraf. La temperatura media anual de la zona es $12{ }^{\circ} \mathrm{C}$ y la precipitación media anual es $1.546 \mathrm{~mm}$. La selección de los árboles se enfocó en representar variabilidad de atributos relacionados con madera estructural como volumen, diámetro de ramas y velocidad acústica.

Los atributos del cuadro 1 son regularmente incluidos en programas de mejora genética de $P$. radiata (Ivković et al. 2006). El DMe es utilizado para clasificar y valorar las trozas y está íntimamente relacionado con el volumen de recuperación de madera aserrada durante el aserrío. El IRa corresponde al diámetro promedio de las cuatro ramas más grandes de cada cuadrante de la troza y tiene un efecto negativo en la rigidez de la madera. Finalmente, el $\mathrm{MoE}_{\mathrm{d}}$ describe la capacidad de un objeto para ser deformado elásticamente, pero no permanentemente, cuando este recibe una fuerza (Chauhan 2006). La ecuación [2] describe su cálculo:

$$
\mathrm{MoE}_{\mathrm{d}}=V^{2} \times D v
$$

Donde: $\mathrm{MoE}_{\mathrm{d}}=$ módulo de elasticidad dinámico $(\mathrm{GPa})$, $V=$ velocidad del sonido $\left(\mathrm{km} \mathrm{s}^{-1}\right)$ registrada con Director HM200 en trozas con corteza, y $D v=$ densidad verde de la madera $\left(\mathrm{kg} \mathrm{m}^{-3}\right)$, la cual fue medida.

El módulo de elasticidad estático de las tablas $\left(\mathrm{MoE}_{\mathrm{e}}\right)$ fue obtenido a través de pruebas mecánicas (machine stress grading). Del cuadro 2 se observa que, en promedio, más del $50 \%$ del volumen comercial de las 71 trozas no califica como estructural, en tanto que la madera estructural, $\mathrm{MoE}_{\mathrm{e}} \geq 8 \mathrm{GPa}$, corresponde al $34 \%$.

La regresión parcial propuesta modeló la relación entre el retorno de conversión de la troza $\left(\mathrm{RC}, \mathrm{US} \$ \mathrm{~m}^{-3}\right)$ y sus atributos. El RC refleja la recuperación de valor las trozas y se calcula como la diferencia entre el ingreso por la venta de la madera producida a partir de $1 \mathrm{~m}^{3}$ de troza, y los costos de procesamiento (Davis et al. 2001). Los productos son madera estructural $\left(\mathrm{MoE}_{\mathrm{e}}>8 \mathrm{GPa}\right)$ con un precio de

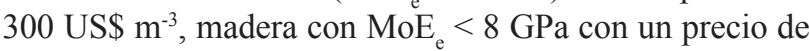

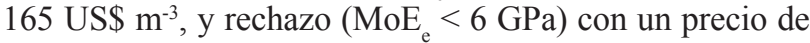


con un precio $20 \%$ superior al estructural, basados en las diferencias relativas observadas en Nueva Zelandia (Alzamora y Apiolaza 2013). El costo de producción se conside-

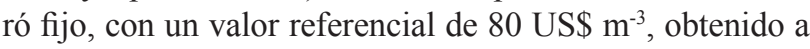
través de consultas a aserraderos de la provincia del Biobío.

Una vez calculados los RC, se procedió a ajustar la regresión parcial según la ecuación [3]:

$$
R C=\beta_{0}+\beta_{1} z_{1}+\beta_{2} z_{2}+\beta_{3} z_{3}+\ldots+\beta_{n} z_{n}
$$

Donde: $\mathrm{RC}=$ retorno de conversión de la troza $\left(\mathrm{US} \$ \mathrm{~m}^{-3}\right.$ troza), $\beta_{i}=$ coeficiente que corresponde al valor económico del atributo $i$, y $z_{i}=$ nivel o magnitud del atributo contenido en cada $\mathrm{m}^{3}$ de troza. 
La regresión parcial fue ajustada por el método de mínimos cuadrados ordinarios usando el paquete estadístico $\mathrm{R}$ (R Development Core Team 2008).

\section{RESULTADOS}

Análisis de correlación permitieron explorar las relaciones entre atributos y RC de las trozas. Así, la correlación entre $\mathrm{MoE}_{\mathrm{d}}$ y DMe fue -0,49 $(P<0,05)$, en tanto que la correlación entre DMe e índice de rama fue $0,48(P<0,05)$. La correlación entre la velocidad acústica y el RC de las trozas fue 0,55 $(P<0,05)$ y las trozas con RC superior a la media $\left(57{\left.\mathrm{US} \$ \mathrm{~m}^{-3}\right)}^{-3}\right.$ generaron lecturas de velocidad superiores a los $2,8 \mathrm{~km} \mathrm{~s}^{-1}$. Así mismo, trozas con lecturas de velocidad $>3 \mathrm{~km} \mathrm{~s}^{-1}$ gene-

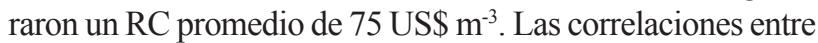
RC y $\mathrm{MoE}_{\mathrm{d}}$, y RC y DMe fueron de $0,83(P<0,05)$ y $-0,27$ $(P<0,05)$, respectivamente. La figura 1 presenta las tendencias observadas entre el RC y los atributos $\mathrm{MoE}_{\mathrm{d}}$ y DMe.

Cuadro 1. Promedio y desviaciones estándar (DE) de los atributos de segunda y tercera trozas.

Mean values and standard deviations (DE) of second and third log attributes.

\begin{tabular}{llcrrr}
\hline & \multirow{2}{*}{$\begin{array}{l}\text { Variable } \\
\text { Media }\end{array}$} & & \multicolumn{2}{c}{ Segunda troza $(\mathrm{N}=35)$} & \multicolumn{2}{c}{ Tercera troza $(\mathrm{N}=36)$} \\
\cline { 3 - 6 } & & \multicolumn{1}{c}{$\mathrm{DE}$} & \multicolumn{1}{c}{ Media } & \multicolumn{1}{c}{$\mathrm{DE}$} & \\
\hline Diámetro menor $(\mathrm{DMe})$ & $\mathrm{cm}$ & 44,91 & 8,41 & 39,77 & 7,71 \\
Módulo de elasticidad dinámico $\left(\mathrm{MoE}_{\mathrm{d}}\right)$ & $\mathrm{GPa}$ & 7,94 & 1,47 & 7,93 & 1,26 \\
Densidad verde $(\mathrm{Dv})$ & $\mathrm{kg} \mathrm{m}^{-3}$ & 906,97 & 66,08 & 919,61 & 63,47 \\
Densidad básica $(\mathrm{Db})$ & $\mathrm{kg} \mathrm{m}^{-3}$ & 382,34 & 28,69 & 377,97 & 28,70 \\
Índice de rama $(\mathrm{IRa})$ & $\mathrm{mm}$ & 4,96 & 1,60 & 5,94 & 1,90 \\
Velocidad acústica $(\mathrm{V})$ & $\mathrm{km} \mathrm{s}^{-1}$ & 2,95 & 0,27 & 2,93 & 0,24 \\
\hline
\end{tabular}

Cuadro 2. Promedio y desviaciones estándar (DE) del volumen de madera por troza $\left(\mathrm{m}^{3} \mathrm{y} \%\right)$, clasificada por $\mathrm{MoE}_{\mathrm{e}}$, a partir de las 71 trozas.

Mean values and standard deviations (DE) of lumber volume per log ( $\mathrm{m}^{3}$ and \%), classified per $\mathrm{MoE}_{\mathrm{e}}$, from $71 \operatorname{logs}$.

\begin{tabular}{ccccccccccc}
\hline & \multicolumn{2}{c}{$\mathrm{MoE}_{\mathrm{e}} 6 \mathrm{GPa}$} & \multicolumn{2}{c}{$\mathrm{MoE}_{\mathrm{e}} 8 \mathrm{GPa}$} & \multicolumn{2}{c}{$\mathrm{MoE}_{\mathrm{e}} 10 \mathrm{GPa}$} & \multicolumn{2}{c}{$\mathrm{MoE}_{\mathrm{e}} 12 \mathrm{GPa}$} & \multicolumn{2}{c}{ Rechazo } \\
\hline & $\mathrm{m}^{3}$ troza $^{-1}$ & $(\%)$ & $\mathrm{m}^{3}$ troza $^{-1}$ & $(\%)$ & $\mathrm{m}^{3}$ troza $^{-1}$ & $(\%)$ & $\mathrm{m}^{3}$ troza $^{-1}$ & $(\%)$ & $\mathrm{m}^{3}$ troza $^{-1}$ & $(\%)$ \\
\hline Media & 0,236 & 51,31 & 0,084 & 19,83 & 0,059 & 15,05 & 0,014 & 3,55 & 0,054 & 10,26 \\
DE & 0,154 & 22,50 & 0,066 & 14,19 & 0,062 & 15,72 & 0,034 & 9,03 & 0,100 & 16,44 \\
\hline
\end{tabular}
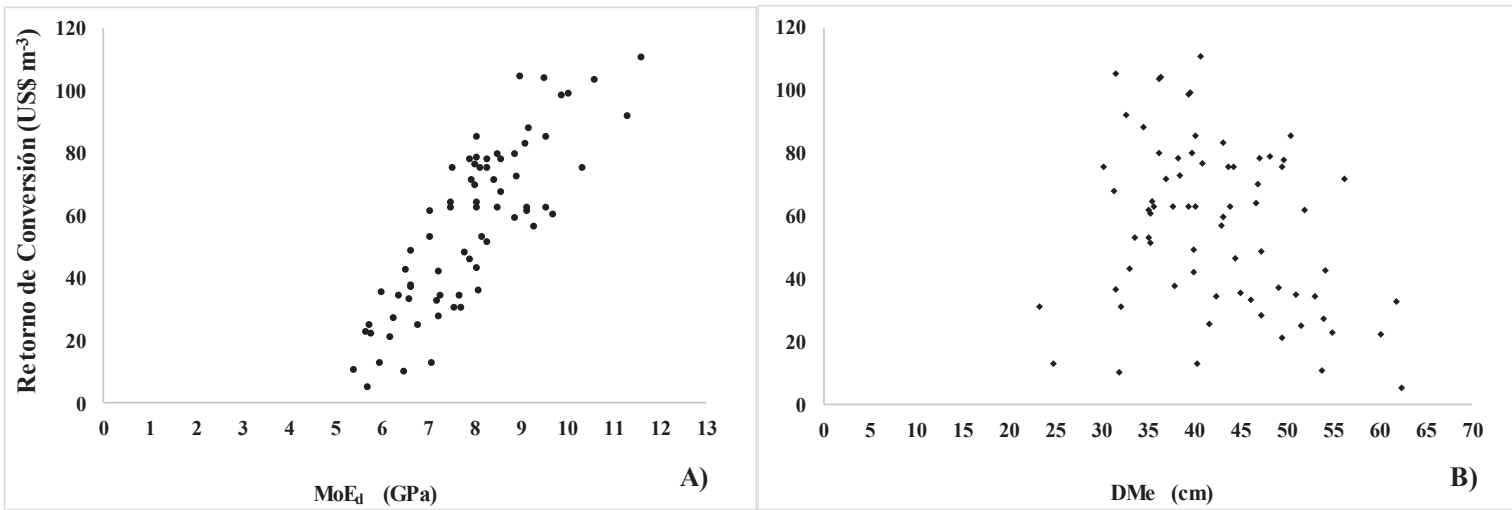

Figura 1. Relación entre A) retorno de conversión y módulo de elasticidad dinámico $\left(\mathrm{MoE}_{\mathrm{d}}\right)$ y $\left.\mathrm{B}\right)$ retorno de conversión y diámetro menor (DMe).

Relationship between A) conversion return and $\mathrm{MoE}_{\mathrm{d}}$, and $\mathrm{B}$ ) conversion return and DMe. 
Los coeficientes de la regresión parcial asociados a $\mathrm{MoE}_{\mathrm{d}}$ y a DMe son positivos y significativos $(P<0,05)$, sin embargo, el IRa resultó no significativo (cuadro 3). Se utilizaron variables dummy para evaluar diferencias de intercepto y pendiente entre segundas y terceras trozas, pero resultaron no significativas $(P>0,05)$; así, la regresión parcial se ajustó considerando las 71 trozas.

El R ${ }^{2}$ ajustado de la regresión parcial fue $0,70(P<0,05)$. Así, el valor económico del $\mathrm{MoE}_{\mathrm{d}}$ resultó ser 17,1 US\$, es decir, que si este atributo aumenta en $1 \mathrm{GPa}$, el RC aumen-

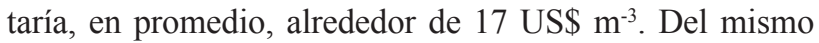
modo, por un aumento de $1 \mathrm{~cm}$ en el DMe, el RC aumenta-

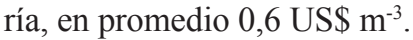

\section{DISCUSIÓN}

El $\mathrm{MoE}_{\mathrm{d}}$ de las segundas y terceras trozas resultó casi idéntico y del orden 7,9 GPa, resultado que no sigue la tendencia reportada por Xu y Walker (2004) quienes indican que el mayor $\mathrm{MoE}_{\mathrm{d}}$ se concentraría en la segunda troza (4 a $8 \mathrm{~m}$ ) para luego decrecer. Por otra parte, siendo tantos los factores que influyen en el comportamiento radial y longitudinal del $\mathrm{MoE}_{\mathrm{d}}$ (ejemplo: edad, heredabilidad, esbeltez del árbol, densidad del rodal, etc.), se considera que estos resultados son válidos y factibles y que son un aporte para ilustrar la variabilidad del $\mathrm{MoE}_{\mathrm{d}}$ en $P$. radiata.

La regresión entregó valores que están en el rango de aquellos reportados por la literatura. Así, los valores del

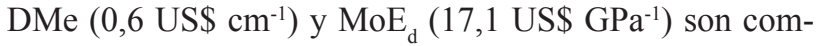
parables a los de otros estudios, guardando las diferencias propias del enfoque y del escenario de costos y precios de cada país. Así, en Nueva Zelandia, Alzamora y Apiolaza (2013) obtienen valores de 1,9 US\$ $\mathrm{cm}^{-1}$ y 14,4 US\$ $\mathrm{GPa}^{-1}$ para DMe y $\mathrm{MoE}_{\mathrm{d}}$, respectivamente, a través de una frontera de eficiencia. Las diferencias se deberían a que la frontera considera un único producto valorado a un precio ponderado por la mezcla de grados estructurales. Con ello, el volumen toma mayor ponderación y como resultado el valor del diámetro menor resulta mayor al de la regresión parcial y el del módulo de elasticidad dinámico menor. En Australia, Ivković et al. (2006) valoraron el $\mathrm{MoE}_{\mathrm{d}}$ en 865 US\$ ha- ${ }^{-1}$ con un modelo bioeconómico en un sistema verticalmente integrado. En el mismo estudio, el valor $\mathrm{MoE}_{d}$ a nivel de aserradero fue 9,3 US\$ GPa ${ }^{-1}$. Las diferencias entre este último y el de la regresión parcial, se deben a que el modelo bioeconómico considera mayores costos de producción. Para el atributo IRa, el coeficiente fue no significativo, y su ponderación económica cero. Alzamora y Apiolaza (2013) reportan lo mismo usando una frontera de eficiencia que modeló la relación entre el volumen de productos $\mathrm{MoE}_{\mathrm{e}} \geq 8 \mathrm{GPa}$, y atributos de troza. Contrariamente, Xu y Walker (2004) indican que este atributo tiene un efecto negativo en la producción de madera estructural. De igual modo, Ivković et al. (2006) reportan un valor de $-1,75$ US $\$ \mathrm{~cm}^{-1}$ para IRa en una muestra de 95 mil trozas, hecho que les permitió acceder a una alta variabilidad del atributo. Así, las diferencias metodológicas entre enfoques y las características de la muestra serían los factores que determinan que el valor de IRa sea negativo o cero. Por ahora no es posible ser concluyente, ya que si bien tal atributo afecta la recuperación de grados estructurales, es debatible que dicho efecto sea siempre significativo en términos económicos.

La regresión parcial fue un enfoque válido para valorar atributos, pero cabe señalar que el método requiere una estructura de precios que refleje las diferencias de calidad del producto (Aubry et al. 1998). Por ello, este estudio debió asumir un precio para los productos con $\mathrm{MoE}_{\mathrm{e}}>10 \mathrm{GPa}$, puesto que en Chile no existe una diferenciación expresa de precios de madera estructural en términos de $\mathrm{MoE}_{\mathrm{e}}$, ya que la clasificación es visual (INN 2006). Sin embargo, una norma mecánica permitiría revelar, con mayor precisión, la calidad estructural de la tabla para promover una mayor especialización de la producción de madera.

La escasa diferenciación precio-calidad también se manifiesta en las trozas, ya que su precio está mayoritariamente basado en forma y volumen (Treolar 2005). Pero al usar un indicador de valor sensible a la calidad como el RC, se observó la alta y directa relación entre este y el $\mathrm{MoE}_{\mathrm{d}}$ y la baja relación con el DMe. Esto, y la alta ponderación económica obtenida para el $\mathrm{MoE}_{\mathrm{d}}$, revelaron la importancia y pertinencia de este atributo para guiar decisiones en manejo, genética y segregación de trozas. Lo anterior es válido incluso para trozas con bajo potencial estructural, como las que se usaron en este estudio. Además, actualmete es posible segregar trozas para calidad estructural a través de

Cuadro 3. Ponderaciones económicas para los atributos $\mathrm{MoE}_{\mathrm{d}}$, DMe e IRa.

Economic weights for attributes $\mathrm{MoE}_{\mathrm{d}}, \mathrm{DMe}$ and IRa.

\begin{tabular}{lccc}
\hline \multicolumn{1}{c}{ Atributo } & Coeficientes & Error estándar & Estadístico t \\
\hline Intercepto & $-98,399^{* * *}$ & 19,981 & $-7,300$ \\
$\mathrm{MoE}_{\mathrm{d}}(\mathrm{GPa})$ & $17,085^{* * *}$ & 1,549 & 10,609 \\
$\mathrm{DMe}(\mathrm{cm})$ & $0,621^{*}$ & 0,245 & 2,002 \\
$\mathrm{IRa}(\mathrm{cm})$ & $-1,2148^{\mathrm{ns}}$ & 1,152 & $-0,591$ \\
\hline
\end{tabular}

$*=P<0,05 ; * *=P<0,01 ; * * *=P<0,001 ; \mathrm{ns}=$ no significativo 
registros de velocidad, porque tal como lo demostró este trabajo, existe una correlación significativa entre velocidad acústica y retorno de conversión de la troza. Dicha tecnología ya se aplica en Australasia (Dickson et al. 2005) y en algunas empresas chilenas, y se espera que se masifique para revelar el perfil de atributos más valorado, y para que en dicho proceso, se generen incentivos de precio que promuevan la producción de trozas de alta calidad.

Futuras investigaciones deberán considerar métodos alternativos para dilucidar el efecto económico de las ramas y evaluar qué enfoque sería el más apto para el escenario de producción de madera estructural en Chile.

\section{AGRADECIMIENTOS}

Este estudio fue financiado por el Proyecto DID S272012 de la Universidad Austral de Chile y apoyado por datos de Solid Wood Innovation (SWI).

\section{REFERENCIAS}

Alzamora RM, LA Apiolaza, DC Evison. 2013. Using a production approach to estimate economic weights for structural attributes of Pinus radiata wood. Scandinavian Journal of Forest Research 28: 282-290.

Apiolaza LA. 2009. Very early selection for wood quality: screening for early winners. Annals of Forest Science 66(6): $1-10$.

Aubry CA, WT Adams, TD Fahey. 1998. Determination of relative economic weights for multitrait selection in coastal Douglasfir. Canadian Journal of Forest Research 28: 1164-1170.

Burdon RD. 1990. Implications of non-linear economic weights for breeding. Theoretical and Applied Genetics 79(1): 65-71.

Chauhan SS, JCF Walker. 2006. Variations in acoustic velocity and density with age, and their interrelationships in radiata pine. Forest Ecology and Management 229: 388-394.
Cotterill PP, N Jackson. 1985. One index selection I. Methods of determining economic weight. Silvae Genetica 34: 2-3.

Davis LS, KN Johnson, PS Bettinger, TE Howard. 2001. Forest Management. 4th Edition. New York, USA. McGraw-Hill. $804 \mathrm{p}$.

Dickson R, B Joe, D Johnstone, S Austin, F Ribton-Turner. 2005. Pre-processing prediction of wood quality in peeler logs grown in northern New South Wales. Australian Forestry 68 (3): 186-191.

Hazel LN. 1943. The genetic basis for constructing selection indexes. Genetics 28: 476-490.

INN (Instituto Nacional de Normalización, CL). 2006. Norma Chilena Oficial 1198/Of2006. Madera- Construcciones en Madera- Cálculo. Santiago, Chile. INN. 213 p.

Ivković M, HX Wu, TA McRae, MB Powell. 2006. Developing breeding objectives for radiata pine structural wood production. I. Bioeconomic model and economic weights. $\mathrm{Ca}$ nadian Journal of Forest Research 36(11): 2920-2931.

Kumar, S. 2004. Genetic parameter estimates for wood stiffness, strength, internal checking, and resin bleeding for radiata pine. Canadian Journal of Forest Research 34(12): 26012610 .

R Development Core Team. 2008. R: a language and environment for statistical computing. R Fundation for Statistical Computing, Vienna, Austria. Disponible en http://www.Rproject.org

Tomlin JA. 1981. A suggested extension of special ordered sets to non-separable non-convex programming problems. NorthHolland Mathematics Studies 59: 359-370.

Treolar C. 2005. Log grade revision: structural log grades. New Zealand Journal of Forestry 49: 33-34.

Xu P, JCF Walker. 2004. Stiffness gradients in radiata pine trees. Wood Science and Technology 38(1): 1-9.

Zhang SY. 1997. Wood quality, its definition, impact and implications for value-added timber management and end uses. In Gosselin TR, G Chauret eds. International Wood Quality Workshop: timber management toward wood quality and end-product value. Quebec, Canada. p. 17-39. 
